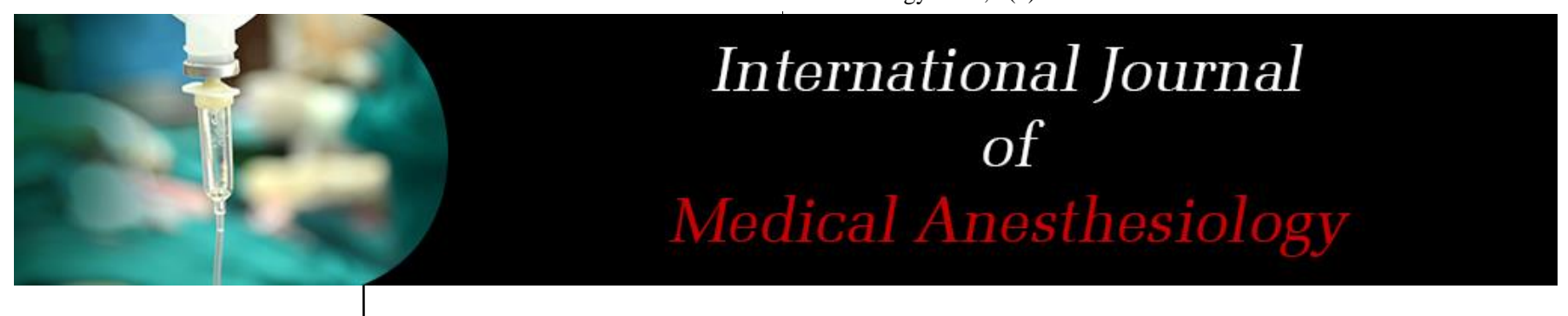

E-ISSN: 2664-3774

P-ISSN: 2664-3766

www.anesthesiologypaper.com

IJMA 2021; 4(2): 07-11

Received: 05-02-2021

Accepted: 08-03-2021

Dr. Tati Parasuram

Junior Consultant,

Department of Anesthesia and

Critical Care, Helius Health

Care Pvt. Ltd., KIMS Saveera

Hospital, Anantapur,

Hyderabad, Telangana, India

Dr. Harish Nadagoudar Assistant Professor,

Department of Anesthesiology, GIMS, Gadag, Karnataka, India
Corresponding Author: Dr. Harish Nadagoudar Assistant Professor,

Department of Anesthesiology, GIMS, Gadag, Karnataka,

India

\section{Comparison of effect of spinal anesthesia on heart rate and blood pressure between hypertensive patients on $\beta$-blockers or calcium channel blockers and normotensive patients: A prospective observational cohort study}

\author{
Dr. Tati Parasuram and Dr. Harish Nadagoudar
}

DOI: https://doi.org/10.33545/26643766.2021.v4.i2a.224

\section{Abstract}

Background: Hypertensive patients develop wide swings in blood pressure intraoperatively, especially after spinal anesthesia. Long term antihypertensive agents can modify this effect by controlling blood pressure. This study was undertaken to evaluate the haemodynamic effect in hypertensive patients on regular treatment with calcium channel blockers and beta-blockers who are undergoing elective surgery under spinal anesthesia and compared with normotensives.

Methodology: 183 patients were included in the study; 61 patients were normotensives (Group A) and 61 patients were hypertensive who were regularly on calcium channel blockers (Group C) and 61 patients on beta-blockers (Group B). Both the groups continued the drug on the day of surgery. The baseline blood pressure and heart rate were recorded. After spinal anesthesia, the blood pressure and heart rate were noted at $2 \mathrm{~min}, 4 \mathrm{~min}, 6 \mathrm{~min}, 8 \mathrm{~min}, 10 \mathrm{~min}, 15 \mathrm{~min}, 20 \mathrm{~min}, 25 \mathrm{~min}, 30 \mathrm{~min}, 45 \mathrm{~min}$, $60 \mathrm{~min}, 75 \mathrm{~min}, 90 \mathrm{~min}, 120 \mathrm{~min}, 150 \mathrm{~min}$, and at $180 \mathrm{~min}$.

Statistical analysis: One way ANOVA with POSTHOC TUKEY test for comparison of the groups and CHI SQUARE test is used for categorical variables. $P<0.05$ was considered as statistically significant.

Results: The hypotension was more in Group C compared to other groups, but is not statistically significant $(P=0.42)$, and requirement of vasopressor to treat hypotension is slightly more in Group $C$ and is not statistically significant $(\mathrm{P}=0.93)$. Bradycardia (heart rate $<50)$ is seen more in Group $\mathrm{B}$ and were statistically significant $(P<0.001)$. The usage atropine to treat bradycardia was more in study group and was statistically significant $(P<0.002)$.

Conclusion: Incidence of hypotension was slightly more with patients on calcium channel blockers which is slightly more than beta blocker and normotensive group. This does not warrant any discontinuation of calcium channel blocker prior to surgery, but incidence of bradycardia was seen more in patients on beta blockers who needed atropine. Anesthesiologists should anticipate and be adequately prepared for any untoward consequences.

Keywords: Hypertension, spinal anesthesia, calcium channel blockers, hypotension, beta blockers, bradycardia

\section{Introduction}

Single injection spinal anesthesia with local anesthetic is most commonly used for surgery to the lower abdomen, pelvic organs and lower limbs. It has various advantages over general anesthesia. Hypotension is the most common complication associated with spinal anesthesia. Though it is seen in almost all the patients, there appears to be a relatively higher incidence in hypertensive patients sometimes causing hemodynamic compromise. Poorly or untreated hypertension over a period of many years doubles the risk of cardiovascular diseases, including coronary heart disease, congestive heart failure, ischemic and hemorrhagic stroke, renal failure and peripheral arterial disease. Hypertensive patients can develop wide swings in blood pressure intraoperatively, which increase the risk of postoperative Cardiac and renal complications such as myocardial ischemia, cerebrovascular accidents and acute renal failure. The cardiovascular effects of neuraxial blocks are similar in some ways to the combined use of intravenous $\alpha-1$ and $\beta$-adrenergic blockers i.e., decreased heart rate and arterial blood pressure ${ }^{[1]}$. The sympathectomy that accompanies the spinal anesthesia 
depends on the level of the block, extending for two to six dermatomes above the sensory level with spinal anesthesia and at the same level with epidural anesthesia. This causes venous and arterial vasodilatation, but because of large amount of blood in the venous system (approximately $75 \%$ of total blood volume) the venodilatation effect predominates whereas the vascular smooth muscle on the arterial side of the circulation retains a considerable degree of autonomous tone. If normal cardiac output is maintained, total peripheral resistance should decrease by $15-18 \%$ in normovolemic healthy patients even with near total sympathectomy. In elderly patients with cardiac disease, systemic vascular resistance may decrease almost $65 \%$, whereas cardiac output decreases only $10 \%$. Heart rate during high neuraxial block typically decreases as a result of blockade of the cardio accelerator fibres arising from $\mathrm{T} 1$ to T4. The heart rate may also decrease as a result of a fall in right atrial filling. A dilemma always existed as to which anti-hypertensive agent has to be stopped before the surgery and which can be continued peri-operatively. Patients with well controlled hypertension should normally continue their medication up to, and including, the day of surgery. There are conflicting reports on the need to continue Angiotensin Converting enzyme Inhibitor on the day of surgery ${ }^{[2,3]}$. It has long been taught that the decrease in blood pressure after neuraxial block can be minimized by administration of crystalloids intravenously by preloading or coloading. When all data are considered, it appears that $12 \mathrm{ml}$ per $\mathrm{kg}$ of body weight hydration regimens appear to temporarily increase preload and cardiac output without consistently increasing arterial pressure or preventing hypotension. A dilemma always existed to which antihypertensive agent has to be stopped before the surgery and which can be continued perioperatively. Studies have been done to determine whether it is beneficial to continue calcium channel blockers and betablockers in patients undergoing general anesthesia. There is inadequate evidence on the effects of calcium channel blockers and beta-blockers on blood pressure in hypertensive patients undergoing spinal anesthesia. The study was undertaken to observe the variation in blood pressure and heart rate, and requirement of ephedrine and atropine for hemodynamic stability in normotensive and hypertensive patients taking antihypertensive medications $\beta$ blockers and calcium channel blockers, and undergoing elective surgery under spinal anesthesia.

\section{Aim and Objectives \\ Aim}

To compare the variations of blood pressure and heart rate during spinal anesthesia in hypertensive patients on calcium channel blockers or beta-blockers and normotensive patients.

\section{Objective}

To compare the incidence of hypotension and bradycardia, and requirement of ephedrine and atropine to maintain hemodynamic stability after spinal anesthesia in hypertensive patients, who are on anti-hypertensive medications i.e., calcium channel blockers or beta-blockers with normotensive patients.

\section{Material and Methods}

The institutional ethics committee approval and written informed consent from patients is obtained.

Study site: Narayana health city, Bommasandra, Bangalore. Study period: $2^{\text {nd }}$ December $2016-31^{\text {st }}$ October 2017.
Study design: Prospective observational cohort study.

Study population: Patients undergoing elective surgeries of lower abdomen, perineum and lower limbs under spinal anesthesia.

\section{Inclusion criteria}

a. Patients with age above 18 years and below 60 years.

b. Patients scheduled for elective surgeries of lower abdomen, lower limbs and perineum under spinal anesthesia.

c. Patients diagnosed to have essential hypertension receiving beta blockers or calcium channel blockers of more than 1 month duration.

d. The patients should have taken the drug pre operatively as per schedule.

e. ASA-PS I \& II.

f. Inclusion criteria for control group

g. All of the above except $\mathrm{c}$ and $\mathrm{d}$.

\section{Exclusion criteria}

a) Known cases of diabetes mellitus.

b) Patients having coronary artery disease, valvular heart disease, and other cardiac diseases.

c) Patients with severe hypovolemia, sepsis.

d) Pregnant women.

e) Patients with BMI > 35 .

f) Patients with ASA-PS III or IV

\section{Methodology}

After ethical committee approval, 183 patients aged between 18 and 60 years of either sex posted for elective lower abdomen, perineum and lower limb, at Narayana health city, Bommasandra, Bengaluru were selected for the study after taking an informed consent. Patients who satisfies with inclusion and exclusion criteria are included in the study. 183 patients are included in the study; 61 patients are normotensives (Group A) and 122 patients are hypertensive who were regularly on calcium channel blockers 61 (Group C) and patients on beta-blockers 61 (Group B) for more than one month duration. Both the groups continued the drug on the day of surgery. At the preanesthetic visit, patients will be assessed, explained about the procedure, anesthesia and consent will be obtained. Each patient is allocated a unique identification number. Detailed history regarding the antihypertensive medications, duration of treatment and other coexistent diseases, and other medications, if any, will be obtained. Airway assessment by Mallampatti grading. Nutritional status, height and weight of the patient. A detailed examination of the cardiovascular system, Respiratory system and Central nervous system. Examination of the spine is done. The following investigations were done in all patients. Haemoglobin estimation, Urine examination for albumin, sugar and microscopy, Standard 12-lead electrocardiogram, Random blood sugar, Blood urea and Serum creatinine. The patients were pre-medicated with tablet alprazolam $0.5 \mathrm{mg}$ and tablet pantoprazole $40 \mathrm{mg}$ orally at night before surgery and they were kept nil orally $6 \mathrm{hrs}$ for solids and $2 \mathrm{hrs}$ for clear fluids. On arrival in the operating room, 18 gauge IV cannula will be inserted in the non-dominant hand. All patients will be coloaded with $20 \mathrm{ml} / \mathrm{kg}$ of Ringer Lactate solution. Standard monitoring included continuous ECG, pulse rate, $\mathrm{SPO}_{2}$ and automated noninvasive blood pressure (systolic blood pressure, diastolic blood pressure and mean arterial pressure) will be carried out. Baseline values will be taken as the reading during rest period. Subarachnoid block will 
be performed in the left lateral position. With all aseptic precautions, local infiltration with $2 \%$ lignocaine $2 \mathrm{CC}$ is infiltrated in the L3-L4 space, using 25 gauge QuinckeBabcock spinal needle, when free flow of CSF is established, $3 \mathrm{ml}$ of $0.5 \%$ hyperbaric bupivacaine will be administered over 10 -second period. The level of sensory blockade will be determined with pinprick at 5 minutes and 15 minutes after the blockade. Non-invasive blood pressure and heart rate will be recorded every 2 minutes for the first 10 minutes and every 5 minutes for 30 minutes and every 15 minutes for 90 minutes and every 30 minutes till 180 minutes.

The patients eligible will be assessed with the following hemodynamic properties during the entire period of the surgery and after the surgery until spinal anesthesia wears off.

a. Blood pressure

b. Heart rate

c. Requirement of ephedrine and atropine to hemodynamically stabilize the patient

Hypotension is defined as a decrease of mean arterial blood pressure of more than $20 \%$ from baseline ${ }^{[8]}$. Maintenance infusion of ringer lactate is continued throughout the surgery. Hypotensive patients are treated with rescue IV boluses of ephedrine $3 \mathrm{mg} /$ dose and IV fluids $5 \mathrm{ml} / \mathrm{kg}$ until both SBP and MAP increased above the threshold level. The time to the first rescue dose and the total dose of ephedrine required is recorded. Bradycardia is defined as heart rate less than 50 beats per minute. Patients with symptomatic bradycardia are treated with injection atropine $0.6 \mathrm{mg} \mathrm{IV}$. Side effects like nausea and vomiting are treated accordingly.

\section{Statistical methods}

Baseline data was analysed using descriptive statistics. The changes of systolic blood pressure, diastolic blood pressure, mean arterial pressure and heart rate were analysed as follows; The changes of the parameter within the group was analyzed using one way ANOVA and the variation within the different time intervals was analyzed with the post-hoc tukey tests. Gender, ASA, Hypotension and other categorical variables were compared between the three groups using chi square tests. 'P value $<0.05$ was considered significant and $<0.01$ highly significant.

\section{Results}

A total of 183 patients who had undergone lower abdominal, perineal and lower limb surgeries under spinal anesthesia as per inclusion criteria were studied over a period of 11 months.

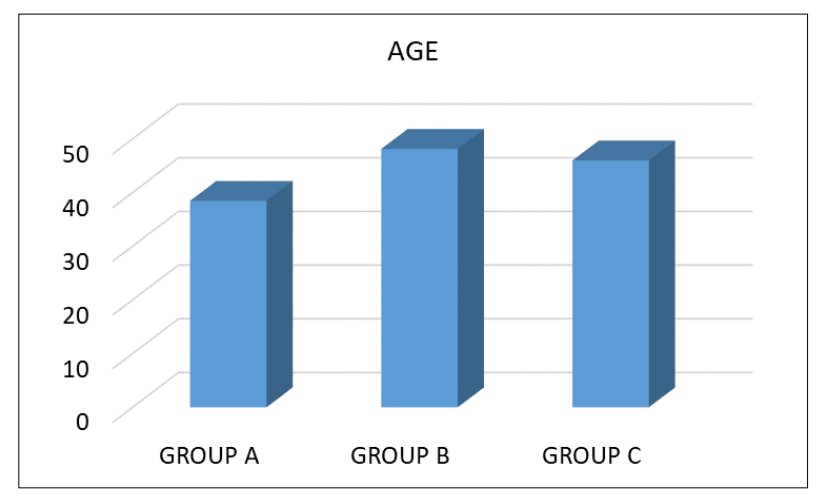

Fig 1: Age distribution
Comparison of AGE between the three groups shows that GROUP B has the highest value of 48.11 and GROUP A has the least value of 38.44 . This difference is statistically Significant with a test value of $37.452 *$ and $\mathrm{p}$ value of $<0.001$.

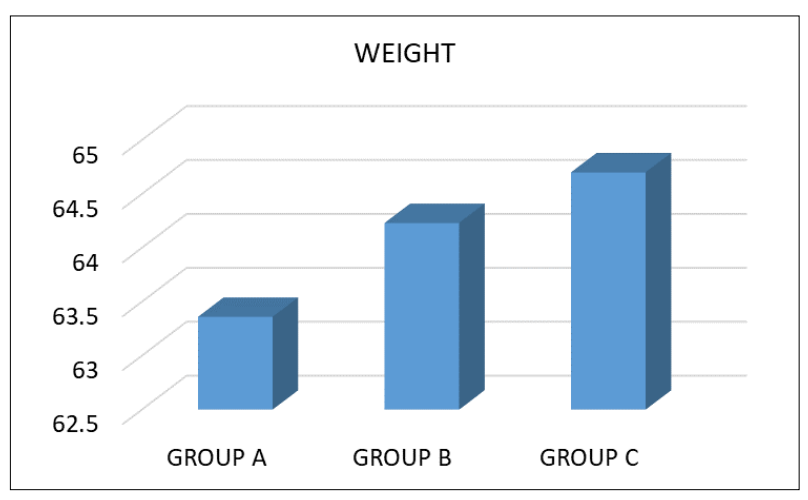

Fig 2: Weight distribution

Comparison of WEIGHT between the three groups shows that GROUP C has the highest value of 64.7 and GROUP A has the least value of 63.36. This difference is statistically Insignificant with a test value of 0.507 and $p$ value of 0.603

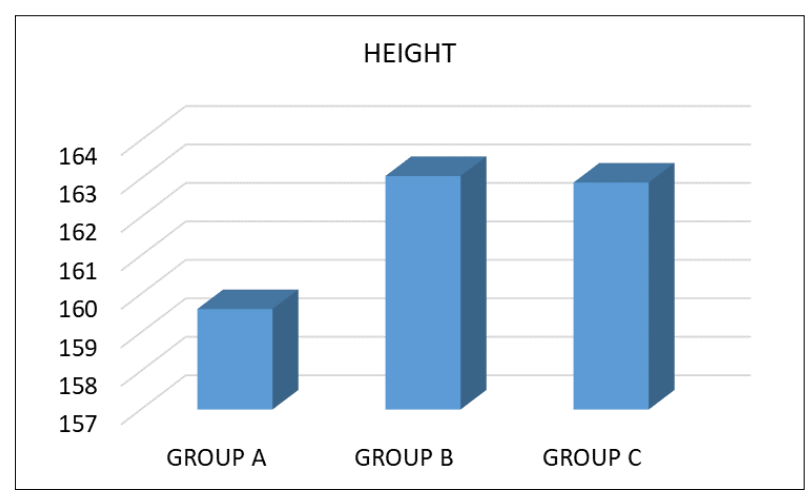

Fig 3: Height distribution

Comparison of HEIGHT between the three groups shows that GROUP B has the highest value of 163.07 and GROUP A has the least value of 159.61.

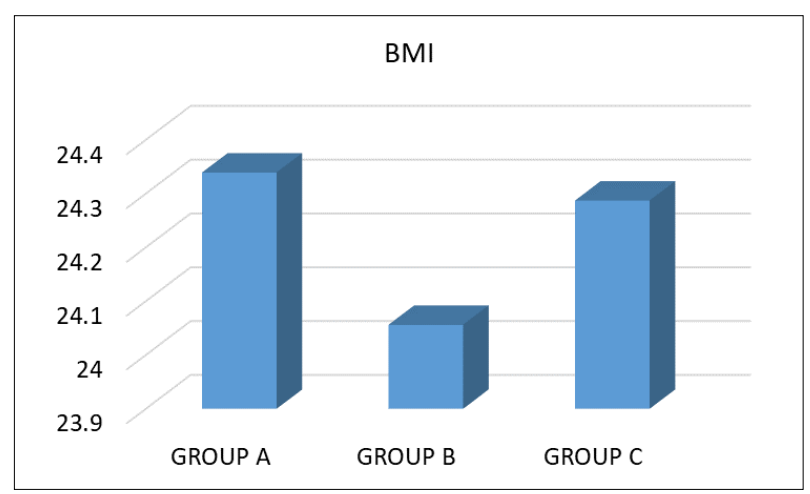

Fig 4: Body mass index

Comparison of BMI between the three groups shows that GROUP A has the highest value of 24.339 and GROUP B has the least value of 24.056. This difference is statistically Insignificant with a test value of 0.316 and $p$ value of 0.73 . 


\section{Discussion}

"Comparison of effect of spinal anesthesia on heart rate and blood pressure between hypertensive patients on beta blockers or calcium channel blockers and normotensive" was undertaken to compare the incidence of hypotension and bradycardia, and requirement of ephedrine and atropine to maintain hemodynamic stability after spinal anesthesia in hypertensive patients, who are on anti-hypertensive medications i.e., calcium channel blockers or beta-blockers with normotensive patients.

\section{Drug selected for the study}

In our study, the drug selected for subarachnoid block was Bupivacaine.

\section{Anti-hypertensive medication}

Patients who are on variable doses of calcium channel blockers (amlodipine 2.5, 5, $10 \mathrm{mg}$ ) (group C) or beta blockers (atenolol 25, 50mg and metoprolol 25 and $50 \mathrm{mg}$ ) (group B) with duration of hypertension more than 1 month are included in group $\mathrm{C}$ and group $\mathrm{B}$ respectively. Control group are devoid of hypertension comprising of ASAI.

\section{Type of surgeries selected for the study}

Patients undergoing elective surgeries of lower abdomen, perineum and lower limb under spinal anesthesia are selected for the study.

\section{Concentrations of the drug selected}

The commonly used concentrations of Bupivacaine for spinal anesthesia is $0.5 \%$. Hyperbaric Bupivacaine is commonly used. Hence we planned to conduct this study with hyperbaric Bupivacaine.

\section{Dose and volume of the drug selected}

In our study we have selected hyperbaric bupivacaine $0.5 \%$ without any adjuvants. The volume used is constant for all the groups i.e., $3 \mathrm{ml}$.

\section{Demographic data}

Demographic data age, weight, height and BMI in all the three groups was comparable. The results were statistically insignificant in weight and BMI. Age of the patients in all the three groups was comparable and come as statistically significant, but we have excluded the age group above $60 \mathrm{yrs}$ who are more prone to hypotension in our study.

\section{Level of sensory blockade}

The level of sensory block attained in T-6 and T-8 in most of the patients. The difference is statistically insignificant between the three groups.

\section{Heart rate}

4 patients in normotensive group, 6 patients in calcium channel group and 51 patients in beta blocker group developed bradycardia (heart rate $<50 \mathrm{bpm}$ ). Which is highly statistically significant. However patients in normotensive group and calcium channel group did not require atropine to treat bradycardia, but 6 patients in beta blocker group required atropine $0.6 \mathrm{mg}$ to treat bradycardia which is statistically significant.

\section{Blood pressure}

Hypotension (MAP < 20\% from baseline) is seen in $44.3 \%$ in group A (27/61), 52.5\% in group B (32/61), and 55.7\% in group C (34/61). Although the incidence of hypotension is more in calcium channel group (group C) $55.7 \%$ it is statistically insignificant $(\mathrm{P}=0.426)$. Requirement of ephedrine to treat hypotension is more in calcium channel group (group C) $28 \%$ when compared with normotensive group (group A) $22.9 \%$ and beta blocker group (group B) $26.3 \%$.

Hypotension is the most frequent side effect associated with spinal anesthesia, predisposing the individual to myocardial and brain ischaemia. Identification of risk factors for prediction of early hypotension were age, female sex, body mass index $>30 \mathrm{~kg} / \mathrm{m} 2$, history of hypertension, diabetes mellitus, anaemia, high baseline heart rate, high systolic and diastolic blood pressure, pulse pressure, sensory level of blockade higher than or equal to T6. Association of hypotension was more in patients with h/o hypertension (OR -1.739) and incidence reduced in patients on anti hypertensives (1.012) ${ }^{[5]}$. Alek Rook et al. ${ }^{[6]}$ found out that an exaggerated decrease in blood pressure occurs in elderly patients with cardiac disease and hypotension was mainly a result of decrease in systemic vascular resistance. Hence the knowledge of risk factors for exaggerated hypotension after spinal anesthesia may help the anesthesiologist in adopting preventive measures to minimize the catastrophe. We chose and compared the hemodynamic changes in hypertensive patients without any other cardiac morbidity on antihypertensive drugs for $>1$ month duration with that of normotensives.

Our study compared Variable doses of calcium channel blocker (Amlodipine 2.5, 5, $10 \mathrm{mg}$ ) (group C), B blocker (Atenolol 25, 50mg and Metoprolol 25, 50) (group B) on Hemodynamics after spinal anesthesia with normotensive patients. Though the incidence of hypotension is more with calcium channel blockers $(55.7 \%)$ when compared with betablocker group (52.5\%) and normotensives (44.3\%) it is statistically insignificant. Compared with previous studies ${ }^{[4,}$ 5] which showed significant hypotension and vasopressor requirement in patients who are on calcium channel blockers, our study shows hypotension and vasopressor requirement is more with calcium channel blockers but is not that significant. Bradycardia is seen predominantly in beta blocker group (93.8\%) with 6 patients requiring atropine to treat bradycardia. Though 4 patients in normotensive group and 6 patients in calcium channel group developed bradycardia, none of them required atropine.

\section{Results}

Demographic data: There was no statistical significance in the demographic data.

Average sensory block: The average height of the block after spinal anesthesia was at the 6th to 8th thoracic level.

Table 1: Average sensory block

\begin{tabular}{|c|c|c|c|c|}
\hline Particular & Group A & Group B & Group C & P value \\
\hline Hypotension & $44.3 \%$ & $52.5 \%$ & $55.7 \%$ & 0.426 \\
\hline Requirement of Ephedrine & $22.9 \%$ & $26.3 \%$ & $28 \%$ & 0.093 \\
\hline Bradycardia & $6.6 \%$ & $93.8 \%$ & $9.8 \%$ & $<0.001$ \\
\hline Requirement of Atropine & $0.0 \%$ & $9.8 \%$ & $0.0 \%$ & 0.002 \\
\hline
\end{tabular}

The hypotension was evident in Group C compared to other groups, but is not statistically significant $(P=0.426)$, and requirement of vasopressor to treat hypotension is slightly 
more in group $\mathrm{C}$ but is not statistically significant $(\mathrm{P}=$ 0.93). Bradycardia (heart rate $<50$ ) is seen more in Group B and were statistically significant $(P<0.001)$. The usage atropine to treat bradycardia was more in study group and was statistically significant $(P<0.002)$.

\section{Conclusion}

Our study showed incidence of hypotension is more in calcium channel group which is slightly more than betablocker and normotensive group. This does not warrant any discontinuation of calcium channel blocker prior to surgery. Bradycardia following spinal anesthesia was more evident in beta blocker group in our study. The knowledge of these risk factors could be useful in increasing vigilance in those patients most at risk for hypotension and bradycardia in allowing timely therapeutic intervention and taking preventive measure to minimize spinal hypotension and bradycardia.

\section{References}

1. Brown DL. Miller's Anesthesia. Philadelphia: Elsevier; Spinal, Epidural and Caudal Anesthesia 2010, P161237.

2. Cozanitis DA. The importance of inhibiting Angiotensin Convertase Inhibitor treatment before spinal anesthesia-a controlled case report. Anesthesiol Reanim 2004;29(1):16-8.

3. Hohne C, Meier L, Boemke W, Kaczmarczyk G. Angiotensin Convertase Inhibitor Inhibitors do not exaggerate the blood pressure decrease in the early phase of spinal anesthesia. Acta Anesthesiologica Scandinavica 2003;47(7):891-6.

4. Chinachoti T, Tritrakarn T. Prospective study of hypotension and bradycardia during spinal anesthesia with bupivacaine: incidence and risk factors. J Med Assoc Thai 2007;90(3):492-501.

5. Singla D, Kathuria S, Singh A et al. Risk factors for development of early hypotension during spinal anesthesia. J Anesthesiol Clin Pharmacol 2006;22(4):387-93.

6. Rooke GA, Freund PR, Jacobson AF. Haemodynamic response and change in organ blood volume during spinal anesthesia in elderly men with cardiac disease. Anaesth Analg 1997;85(1):99-105. 